\title{
ق̈FFRSETI
}

Forseti. Revista de Derecho. Edición de aniversario, Lima, DERUP, 2018, pp 162-174

\section{La defensa del Jurel en la OROP del Pacífico Sur}

\author{
Richard Inurritegui* \\ Milagros Mutsios
}

\begin{abstract}
Resumen.- El aprovechamiento del recurso pesquero depende de la propia naturaleza de los mares y las características migratorias de los peces. Así las cosas, la división de intereses entre Estados costeros y ribereños con Estados que pescan a distancia, ha marcado la pauta de la regulación internacional en relación con el aprovechamiento de este recurso natural. En este contexto, el presente artículo trata de explicar brevemente el contexto de regulación internacional relacionado con la pesca en altamar. Ello con la finalidad de contextualizar la defensa exitosa del Perú por mantener la soberanía en sus aguas jurisdiccionales, en el marco de los acuerdos que dieron origen a la Organización Regional de Ordenamiento Pesquero del Pacífico Sur.
\end{abstract}

Abstract.- The exploitation of the fishing resource depends on the nature of the seas and the migratory characteristics of fishes. Thus, the division of interests between coastal States and coastal States with States that fish at a distance, has set the pattern of international regulation in relation to the use of this natural resource. In this context, this article tries to briefly explain the context of international regulation related to the fishing industry on the high seas. This in order to contextualize the successful defense of Peru to maintain sovereignty in its jurisdictional waters, within the framework of the agreements that gave rise to the South Pacific Regional Fisheries Management Organization.

Abogado por la Pontificia Universidad Católica del Perú. Consultor del Estudio Rodrigo, Elías \& Medrano.

** Bachiller por la Pontificia Universidad Católica del Perú. Asociada del Estudio Rodrigo, Elías \& Medrano. 
El pasado enero se celebró en Lima la Sexta Reunión de la Comisión de la Organización Regional de Ordenamiento Pesquero del Pacífico Sur («OROP-PS») $)^{1}$. Cabe resaltar que previas a estas seis reuniones de Comisión, existieron otras ocho reuniones preparatorias en las que se negoció el texto de lo que sería finalmente la «Convención de Conservación y Manejo de Recursos Pesqueros de Alta Mar en Pacífico Sur» y lo que dio finalmente la partida de nacimiento de esta organización. Así las cosas, el presente artículo busca definir el alcance de esta OROP-PS, los principales temas que regula y la defensa de Perú sobre un recurso pesquero de gran importancia: el jurel.

En el año 2006, Chile, Australia y Nueva Zelanda, iniciaron procesos de consulta para buscar mecanismos de cooperación entre Estados en la conservación y manejo de especies transzonales y la protección a la biodiversidad. Estos procesos derivaron en lo que es, en la actualidad, la OROP-PS.

Cierto es que la presencia de Chile en estos procesos de consulta, se debió a su preocupación por la sostenibilidad de su pesquería de jurel (la más importante para ellos) como consecuencia de la pesca indiscriminada que hacía la flota extranjera de los llamados Estados pesqueros a distancia que operaban en la alta mar (el stock del jurel que pesca Chile tiene un rango amplio de recorrido que se adentra en la alta mar allende a su costa y; por tanto estaba a disposición de dicha flota extranjera).

En este escenario, cabe preguntar, ¿qué llevó a Chile a buscar ayuda en países tan alejados? los que, si bien son del Pacífico, están del otro lado.

Durante años intentó que los países vecinos de este lado del Pacífico Sur (i.e. Perú, Ecuador y Colombia), con los que integra la llamada Comisión Permanente del Pacífico Sur, implementaran estrictas medidas de acceso a los puertos que impidieran o dificultaran la operación de flota extranjera que hacía pesca ilegal, no declarada o no reportada (INDNR por sus siglas en inglés), la que consideraban, perjudicaba seriamente la sostenibilidad de su stock de jurel.

Como finalmente no hubo una homologación de estas medidas, buscaron una manera distinta de impedir la pesca indiscriminada de jurel en la alta mar y eso los llevó a buscar a Australia y Nueva Zelanda. Así comienza la historia de esta OROP-PS.

\footnotetext{
SPRFMO, por su definición en inglés (South Pacific Regional Fisheries Management Organisation). En la actualidad cuenta con 15 Estados miembros y 4 Partes Cooperantes No Contratantes.
} 


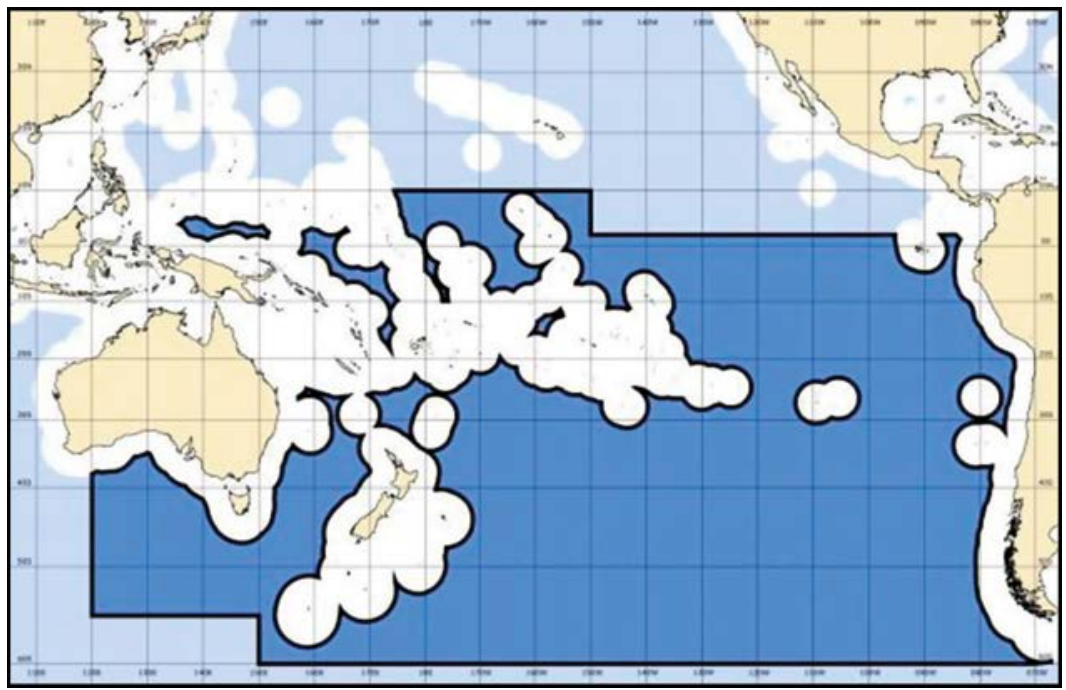

La OROP-PS ha publicado un mapa que describe el área de aplicación conforme a los términos legales en el artículo 5 de la Convención, se precisa que el mismo sólo tiene fines informativos, no tiene estatus legal y no forma parte del texto de la Convención ${ }^{2}$.

Buscando ser didáctico, este artículo se divide en dos partes. La primera busca acercar al lector al ordenamiento jurídico internacional para el manejo de los recursos pesqueros de alta mar, con énfasis en la formación de OROP's, como la que trata este artículo.

Con dicho contexto, la segunda parte describe la actuación de Perú en esta organización, el trabajo conjunto entre sector público y privado, así como la defensa de sus derechos como Estado costero frente a los intereses de los Estados pesqueros a distancia y frente a Chile, que tuvo en esta negociación una posición distinta a la que se esperaría de un Estado ribereño o costero. Todo esto para defender nuestro stock de jurel y de los siguientes recursos pesqueros que serán regulados por esta OROP-PS.

\section{Contexto general: la regulación de la pesca en altamar}

Atendiendo al objetivo del presente artículo, es importante que el lector parta de la premisa que, los intereses entre los llamados Estados ribereños o costeros y los Estados pesqueros a distancia, pueden ser en gran medida contrapuestos. En la mayoría de los casos, los Estados pesqueros a distancia son también Estados ribereños o costeros de algún lugar del planeta, con recursos pesqueros en sus aguas jurisdiccionales que han deprimido o depredado y/o han desarrollado además una flota pesquera de gran escala para operar a distancia.

2 Disponible en: http://www.produce.gob.pe/index.php/sobre-el-orop 
En ese contexto de intereses contrapuestos por la pesca en alta mar y tomando en la migración de los recursos pesqueros, los recursos altamente migratorios y los transzonales -los cuales pueden fluctuar entre las aguas jurisdiccionales y las de alta mar- se puede entender la necesidad de los Estados por llegar a acuerdos internacionales que permitan proteger los recursos marinos y evitar su depredación.

Considerando lo anterior, tres (3) regulaciones resultan relevantes al momento de analizar la pesca en la altamar y a efectos de analizar el alcance de la OROP-PS: (i) la Convención de las Naciones Unidas sobre el Derecho del Mar («CONVEMAR»), (ii) Acuerdo sobre la aplicación de las disposiciones de la CONVEMAR («Acuerdo de Nueva York»); y (iii) la Convención para la Conservación y Ordenamiento de los Recursos Pesqueros de Alta Mar del Océano Pacífico Sur («Convención»).

Sobre la CONVEMAR, ésta fue resultado de la Tercera Conferencia sobre el Derecho del Mar la cual inició en 1973 y culminó en 1983. Atendiendo al preámbulo de este documento es posible determinar entre sus objetivos estaba el (i) crear conciencia respecto de los problemas que ocasionaban la falta de regulación de los espacios marítimos, entre los países; y (ii) la necesidad de equilibrar los requerimientos de los países en desarrollo, sean ribereños o sin litoral.

Así las cosas, uno de los aportes más importantes de la CONVEMAR fue el establecimiento de la Zona Económica Exclusiva («ZEE»). Al respecto, el artículo 55 regula lo siguiente:

\begin{abstract}
Artículo 55
Régimen Jurídico específico de la zona económica exclusiva

La zona económica exclusiva es un área situada más allá del mar territorial y adyacente a éste, sujeta al régimen jurídico específico establecido en esta Parte, de acuerdo con el cual los derechos y la jurisdicción del Estado ribereño y los derechos y libertades de los demás Estados se rigen por las disposiciones pertinentes de esta Convención (énfasis nuestro).
\end{abstract}

Sobre la extensión de esta zona, el artículo 57 precisó que se extiende más allá de 200 millas marinas contadas desde las líneas de base a partir de las cuales se mide la extensión del mar territorial ${ }^{3}$. El estable-

\footnotetext{
3 Téngase en cuenta que, el 18 de agosto de 1952, Chile, Ecuador y Perú adoptaron la Declaración sobre Zona Marítima en la que se dispuso, entre otros, «(...) los Gobiernos de Chile, Ecuador y Perú proclaman como norma de su política internacional marítima, la soberanía y jurisdicción exclusivas que a cada uno de ellos corresponde sobre el mar
} 
cimiento de esta zona y su distancia evidentemente convirtió a zonas que podían ser consideradas como «alta mar» en zonas ribereñas; $y$, por ende, de soberanía de Estados ribereños o costeros. Sin embargo, es difícil pretender que los recursos marítimos tomen cuenta de esta división, por lo que se encuentran especies transzonales que migraban entre una zona y la otra.

En respuesta a esta situación, no cabe duda que los Estados pesqueros a distancia que operan en alta mar, intensificaron su accionar al ver recortados sus recursos. Ello, sin lugar a duda, generaba una preocupación para los Estados ribereños o costeros respecto de la excesiva pesca en alta mar de recursos pesqueros transzonales y altamente migratoria.

Así las cosas, el accionar de los Estados pesqueros a distancia desvirtuaba los objetivos de conservación y los derechos regulados a favor de los Estados ribereños o costeros en la CONVEMAR (artículo 56) ${ }^{4}$. Como respuesta, se buscaron soluciones a través de negociaciones con el objetivo de establecer directrices para la pesca en el área fuera de la ZEE con la finalidad de evitar el deterioro de las especies transzonales y altamente migratorias, lo cual también podría generar consecuencias sobre el nivel de recursos marinos en los Estados ribereños ${ }^{5}$.

De esta manera, se desarrollaron diversas conferencias y acuerdos entre los Estados. Como resultado de tales reuniones se aprobó el Acuerdo de Nueva York, el 4 de diciembre de 1995 (artículo 1 del citado Acuerdo). El objetivo principal fue establecer las pautas relativas a la conservación y ordenación de poblaciones de peces transzonales ${ }^{6} \mathrm{y}$ altamente migratorios ${ }^{7}$, mediante la aplicación efectiva de las disposiciones de la CONVEMAR.

que baña las costas de sus respectivos países, hasta una distancia mínima de $\mathbf{2 0 0}$ millas marinas desde las referidas costas (énfasis agregado)». El análisis sobre la falta de definición en la forma de medición de las mencionadas millas no es materia del presente artículo.

4 VILLALOBOS, Jorge. «El régimen de pesca en la Convención de las Naciones Unidas sobre el Derecho del Mar de 1982». En: Derecho del Mar. Análisis de la Convención de 1982. Lima, PUCP, 2001, pp. 186-187.

5 COUVE, Andrés. «Itinerario de la negociación en Naciones Unidas sobre poblaciones de peces transzonales y altamente migratorias». En Pacífico Sur, 22, Lima, Comisión Permanente del Pacífico Sur, 1996, p. 123.

6 Sobre las especies transzonales, el numeral 2 del artículo 63 de la CONVEMAR reguló que «(c)uando tanto en la zona económica exclusiva como en un área más allá de ésta y adyacente a ella se encuentren la misma población o poblaciones de especies asociados, (...)».

$7 \mathrm{Al}$ respecto, el artículo 84 de la CONVEMAR ha establecido lo siguiente: «1. El Estado ribereño y los otros Estados cuyos nacionales pesquen en la región las especies altamente migratorias enumeradas en el Anexo I cooperan, directamente o por conducto de las organizaciones internacionales, apropiadas (...)». 
La doctrina ha determinado cuatro (4) aspectos esenciales relacionados con el Acuerdo de Nueva York: (i) la conservación y ordenamiento de las poblaciones de peces, (ii) mecanismos de cooperación internacional, (iii) seguimiento, control y vigilancia; y (iv) necesidades de los Estados en desarrollo ${ }^{8}$.

En este contexto, importa analizar el artículo 5 del Acuerdo de Nueva York mediante el cual se regula que, los principios generales que deben seguir los Estados (ribereños y pesqueros a distancia), entre ellos los siguientes:

\section{Artículo 5}

A fin de conservar y ordenar las poblaciones de peces transzonales y las poblaciones de peces altamente migratorios, los Estados ribereños o costeros y los Estados que pescan en alta mar, deberán al dar cumplimiento a su deber de cooperar de conformidad con la Convención.

a) Adoptar medidas para asegurar la supervivencia a largo plazo de las poblaciones de peces transzonales y las poblaciones de peces altamente migratorios, los Estados ribereños y los Estados que pescan en alta mar, deberán, al dar cumplimiento a su deber de cooperar de conformidad con la Convención.

(...)

e) Adoptar, en caso necesario, medidas para la conservación y ordenación de las especies que pertenecen al mismo ecosistema o que son dependientes de las poblaciones objeto de la pesca o están asociadas con ellas, con miras a preservar o restablecer tales poblaciones por encima de los niveles en que su reproducción pueda verse gravemente amenazada (énfasis nuestro).

Considerando lo citado, es claro que la finalidad de las medidas es asegurar la sostenibilidad de los recursos pesqueros para lograr su aprovechamiento eficiente.

Adicionalmente, el artículo 7 reguló la compatibilidad de las medidas de conservación y de ordenación:

(...) sin perjuicio de los derechos de soberanía que la Convención reconoce a los Estados ribereños con respecto a la exploración y explotación, la conservación y la ordenación de los recursos marinos vivos dentro de las zonas que se encuentran bajo su jurisdicción nacional, y del derecho de todos los Estados a que sus nacionales se dediquen a la pesca en alta mar de conformidad con la Convención (énfasis nuestro).

8 RAMOS, Eduardo. «El régimen jurídico internacional de la pesca en alta mar y la OROP Del Pacífico Sur». En Agenda Internacional. Año XX, N. 31. Lima, 2013, pp. 71-97. 
Aunque el Acuerdo de Nueva York reguló la no afectación de la soberanía de los Estados ribereños o costeros, el numeral 2 del mencionado artículo establece que:

(...) las medidas de conservación y ordenación que se establezcan para la alta mar y las que se adopten para las zonas que se encuentran bajo jurisdicción nacional habrán de ser compatibles, a fin de asegurar la conservación y ordenación de las poblaciones de peces transzonales y las poblaciones de peces altamente migratorios en general. Con este fin, los Estados ribereños y los Estados que pesquen en alta mar tienen la obligación de cooperar para lograr medidas compatibles con respecto a dichas poblaciones (énfasis nuestro).

A partir de lo anterior es posible concluir que, si bien el Acuerdo de Nueva York reguló que se respetaría la soberanía de los Estados ribereños o costeros, el hecho de determinar que las medidas de manejo de los recursos naturales deben ser «compatibles» con las medidas que los Estados adopten para la pesca en alta mar representa una limitación a tal soberanía presuntamente protegida.

Ello ha llevado a autores como Iglesias a afirmar que el Acuerdo de Nueva York parece orientado a beneficiar los intereses de los Estados que pescan en alta mar y no a los ribereños ${ }^{9}$. Sin embargo, siguiendo a Orrego es posible concluir que, el hecho que se indique que las medidas deben ser «compatibles» no significa que deben ser «iguales», con lo cual no hay una verdadera afectación a la soberanía interna de ningún país ribereño ${ }^{10}$.

Sin lugar a dudas es razonable que exista una suerte de compatibilidad entre las medidas a adoptar respecto de las especies materia de desarrollo en el Acuerdo de Nueva York, pues su sobre-explotación, en la ZEE tiene efectos en alta mar y viceversa, el problema surge cuando hay medidas distintas sobre un mismo objeto y hay que determinar cuál de las dos primaría.

Finalmente, enfatizamos que a partir del Acuerdo de Nueva York se impulsó la cooperación de los Estados para la conservación y la ordenación pesquera. Al respecto, el artículo 8 del citado Acuerdo esta-

9 IGLESIAS, Marta. «Aspectos jurídicos del Acuerdo de Nueva York de 1995 sobre especies transzonales y altamente migratorios». En: Revista Galega de Económica. Vol. 18, núm. 1 2009, pp-1-17.

10 ORREGO, Francisco. «El régimen de la pesca en alta mar y los derechos e intereses del estado ribereño». En: Los cincuenta años de la tesis chilena de las doscientas millas marinas (1947-1997). Santiago de Chile, Universidad de Chile. 1998, p. 109. 
blece que los Estados deberán cooperar entre sí, directamente o por conducto de organizaciones o arreglos. Así las cosas,

(...) en los casos en que una organización o un arreglo subregional o regional de ordenación pesquera tenga competencia para establecer medidas de conservación y ordenación respecto de determinadas poblaciones de peces transzonales o poblaciones de peces altamente migratorios, los Estados (...) cumplirán su obligación de cooperar haciéndose miembros de la organización o participantes en el arreglo, o comprometiéndose a aplicar las medidas (...) (énfasis nuestro).

Tomando en cuenta esta obligación de cooperar es que se concluye que, es en el marco de los mandatos del Acuerdo de Nueva York con relación a la cooperación internacional entre Estados ribereños y aquellos de pesca en alta mar para la conservación de peces transzonales o de alta migración que nace la OROP-PS y con ella la Convención.

Producto de las ocho (8) reuniones preparatorias, se creó la OROP-PS en virtud de la Convención. El artículo 2 de este instrumento determina que su objetivo es «(...) garantizar la conservación a largo plazo y el uso sostenible de los recursos pesqueros a través de la aplicación del criterio de precaución y del enfoque basado en los ecosistemas, para salvaguardar de este modo los ecosistemas marinos que albergan dichos recursos (énfasis nuestro)».

A partir de ello, el artículo 4 de la Convención profundiza el concepto de compatibilidad de las medidas de conservación y ordenamiento -tema que ya había sido abordado por el Acuerdo de Nueva York-, señalando que:

\section{Artículo 4}

\section{$(\ldots)$}

2. Las medidas de conservación y ordenamiento que se establezcan para las zonas de alta mar y las que se adopten para las zonas sujetas a jurisdicción nacional habrán de ser compatibles, a fin de asegurar la conservación y ordenamiento de los recursos pesqueros transzonales en su integridad. Las Partes Contratantes, al elaborar medidas de conservación y ordenamiento compatibles entre sí para los recursos pesqueros transzonales, deberán:

(a) tener en cuenta la unidad biológica y demás características biológicas de los recursos pesqueros y las relaciones entre la distribución de los recursos, las actividades pesqueras ejercidas con respecto a tales recursos y las particularidades geográficas de la región de que se trate, así como el grado en que los recursos pesqueros estén presentes y sean objeto de pesca en las zonas sujetas a jurisdicción 
nacional; (b) tener en cuenta el grado de dependencia de los recursos pesqueros de que se trate tanto de los Estados ribereños como de los Estados que realizan sus faenas de pesca en alta mar;(c) garantizar que tales medidas no tengan efectos perjudiciales en el conjunto de los recursos marinos vivos del Área de la Convención (énfasis nuestro).

Sobre este artículo, se ha afirmado que:

Los Estados de pesca distante postulan que, en virtud de la compatibilidad de medidas, las decisiones para la conservación y ordenación adoptadas para la alta mar pueden ser aplicadas en las aguas jurisdiccionales de un Estado ribereño; no obstante, en la Convemar se establece que los Estados ribereños tienen opción preferente para el dictado de las medidas y su traslado a la zona de la alta mar, es decir, administrar los recursos pesqueros transzonales en sus aguas jurisdiccionales ${ }^{11}$.

A partir de lo anterior, queda claro que, si bien mediante el Acuerdo de Nueva York podía afirmarse que no existía una trasgresión a la soberanía de un Estado, el detalle del alcance de tal artículo desarrollado por la Convención, permite sustentar lo contrario. Ello, debido a que se busca limitar la soberanía de un Estado a la situación de toda la zona en donde se pueda ubicar el recurso transzonal, independientemente de si ocupa la ZEE o altamar.

Como veremos y analizaremos en la sección siguiente, esta definición de compatibilidad y la manera cómo se pretendió definir el stock y rango del recurso pesquero (en específico el jurel) será la razón medular que definió la posición de Perú en las negociaciones de la Convención y lo que al final logró conseguir en solitario.

Finalmente, habiendo dejado claro este tema de posibles intereses contrapuestos, pasaremos a desarrollar la actuación de Perú en las negociaciones de esta OROP-PS.

\section{El Perú en la OROP-PS}

Consideramos importante en primer lugar, señalar qué dice la normativa nacional en relación a la regulación de la alta mar y la compatibilidad de medidas, dado que el Perú no es signatario de la CONVEMAR ni del Acuerdo de Nueva York.

11 RAMOS, Eduardo. «El régimen jurídico internacional de la pesca en alta mar y la OROP Del Pacífico Sur». En: Agenda Internacional. Año XX, Nº. 31. Lima, 2013, p. 95. 
Al respecto, resulta relevante mencionar que el artículo 54 de la Constitución Política del Perú establece que:

Artículo 54.- (...) El dominio marítimo del Estado comprende el mar adyacente a sus costas, así como su lecho y subsuelo, hasta la distancia de doscientas millas marinas medidas desde las líneas de base que establece la ley. En su dominio marítimo, el Estado ejerce soberanía y jurisdicción, sin perjuicio de las libertades de comunicación internacional, de acuerdo con la ley y con los tratados ratificados por el Estado (énfasis nuestro).

En esta línea, y atendiendo a lo discutido en el marco de la regulación internacional, el artículo 7 de la Ley General de Pesca regula lo siguiente:

Artículo 7.- Las normas adoptadas por el Estado para asegurar la conservación y racional explotación de los recursos hidrobiológicos en aguas jurisdiccionales, podrán aplicarse más allá de las $\mathbf{2 0 0}$ millas marinas, a aquellos recursos multizonales que migran hacia aguas adyacentes o que proceden de éstas hacia el litoral por su asociación alimentaria con otros recursos marinos o por corresponder a hábitats de reproducción o crianza.

El Perú propiciará la adopción de acuerdos y mecanismos internacionales a fin de procurar el cumplimiento de tales normas por otros Estados, con sujeción a los principios de la pesca responsable (énfasis nuestro).

Tomando en cuenta los artículos citados se concluye que la posición del Perú, reflejada en su regulación interna supone una posible aplicación más allá de las 200 millas, de la compatibilidad de las medidas que como Estado ribereño o costero determina y aplica para las especies transzonales, pero, todo ello, en armonía con el derecho internacional pues propicia la adopción de acuerdos internacionales como son las OROP en general.

El Perú no participó de las iniciales reuniones preparatorias de la OROP-PS que iniciaron desde el 2006 si no hasta el siguiente año, pero inicialmente sin mayor institucionalidad ni mayor coordinación con los grupos de interés en el país.

El sector privado representado por la Sociedad Nacional de Pesquería-SNP, recién empezó su participación en el año 2007 en la reunión en Reñaca, Chile. Como anécdota, contaremos que esa primera representación se dio de manera casi casual y sin que la SNP tuviera claro aún la importancia de lo que se estaba discutiendo en esas reuniones y lo que se pretendía aprobar. A una directora de la SNP que se encontraba en Noruega por razones de trabajo, se le pidió que de 
regreso a Perú pasara por Chile a esta reunión en Reñaca pero sin saber muy bien de qué trataba (la Cancillería le había sugerido a la SNP que participara de esta reunión).

Luego de esta participación, el sector privado fue tomando conciencia de la importancia de lo que se discutía y de lo que estaba en juego (i.e. la soberanía marítima del Perú y el stock del jurel en primera instancia). A partir de ese momento, la SNP tomó una participación activa y, con el apoyo del Ministerio de la Producción-PRODUCE, del Instituto del Mar del Perú-IMARPE, de la Dirección General de Capitanías y Guardacostas de la Marina de Guerra del Perú-DICAPI y del Ministerio de Relaciones Exteriores, se logró armar un grupo de trabajo intersectorial e interinstitucional de coordinación para las futuras reuniones preparatorias de la OROP-PS. Este grupo que, representaba los intereses del Estado peruano, definía la posición peruana que se defendería en las reuniones de la OROP-PS, previamente.

A partir de allí, la participación de la delegación peruana no solo fue activa sino también numerosa y esto, en razón de haber tomado conciencia de lo que estaba en juego para el futuro de nuestra pesquería de jurel y de los siguientes recursos transzonales que se iban a regular en el marco de esta OROP-PS.

Pero si todos los países supuestamente compartían la necesidad de regular la pesquería del jurel en alta mar para velar por su sostenibilidad, era cierto también que los intereses de los Estados países en la pesquería de este recurso, determinarían que del resultado de las negociaciones hubiese quienes ganaban y perdían en mayor o menor medida.

La primera gran discusión giró en torno al stock del jurel y el número de éstos en relación al rango de su desplazamiento. El Perú sostenía, con fundamento científico y técnico, que habían por lo menos dos stocks diferenciados (uno en la ZEE del Perú y su alta mar allende y otro en las de Chile y su alta mar). En base a ello, reclamaba una diferenciación en su estudio, análisis y adopción de medidas.

Los demás países (salvo Rusia que apoyaba la posición peruana y señalaba que había hasta cuatro stocks distintos), sostenían y reclamaban la regulación en función a un stock como unidad pasible de una única regulación. Este primer tema tenía un impacto directo sobre la nuestra pesquería de jurel, pues siempre habíamos tenido soberanía en la determinación de nuestra cuota de pesca y las medidas de manejo a tomar. 
Un segundo punto de relevancia fue la manera de determinar los porcentajes de pesca de las cuotas que se determinen anualmente. Allí el consenso de los otros países era usar el récord de la pesca histórica de las embarcaciones que tradicionalmente hubiesen pescado en la alta mar del Pacífico Sur y, en ese contexto, el Perú estaba en clara desventaja pues no tenía flota pesquera de jurel para la alta mar. En ese punto se logró establecer una ventana de dos años para acreditar flota en el área del OROP-PS y con ello, el Perú pudo inscribir a su flota de cerco con permiso de pesca para jurel y otras tantas embarcaciones de arrastre que tienen mayor rango de acción.

El tema sin embargo de mayor relevancia lo constituyó el ámbito de acción y jurisdicción de la OROP-PS. Conforme a lo explicado en la primera parte de este artículo, este tipo de organizaciones son claramente para regular la pesca de alta mar únicamente y en lo que respecta a las áreas de jurisdicción nacional, la compatibilidad de medidas garantizaba que no hubiese manejos contradictorios en estas áreas y el alta mar.

A pesar de la claridad del ámbito de las OROP's, la intención de los países siempre fue el regular el stock del jurel en lo que llamaban «todo el rango de acción» y ese concepto, incluía las áreas de jurisdicción nacional, lo que constituía una clara violación a la soberanía de los Estados ribereños o costeros. En este punto, se esperaba que los países costeros de esta parte del Pacífico, cerraran filas y exigieran una claridad normativa en la Convención de que su regulación estaba circunscrita a alta mar. Aquí lamentablemente, el Perú se quedó solo.

Ecuador y Colombia nunca tuvieron una actuación protagónica en esta OROP-PS y Chile, de quien se esperaba que como Estado ribereño o costero tuviera una posición similar a la peruana, se alineó con la posición de los otros países por una conveniencia estratégica del stock de jurel que se ubicaba en sus aguas jurisdiccionales y su alta mar allende y la posibilidad de incluir en la pesca histórica para el reparto de los porcentajes de las cuotas, la pesca que históricamente había realizado en sus aguas jurisdiccionales.

En este punto radica el mayor logro de la delegación peruana que durante años participó en las reuniones preparatorias a partir de mantener una solitaria posición principista de no ceder su soberanía en sus aguas jurisdiccionales, aunque esto le significase una menor cuota en alta mar.

En ese contexto y a pesar del consenso de resto de países, se logró la inclusión de un consentimiento expreso del Estado ribereño o costero, 
para que el esfuerzo pesquero y la cuota que se determine, aplique en sus aguas jurisdiccionales. Esta fórmula se recogió en el texto de la Convención de la OROP-PS de la siguiente manera en el artículo 20 sobre «Medidas de Conservación y Manejo»:

Artículo 20, numeral 4, literal a)

Para un recurso pesquero que traspase el área de la Convención y un área bajo jurisdicción nacional de un Estado costero parte o partes:

i) La Comisión debe establecer una cuota total de captura o un esfuerzo pesquero máximo permisible y otras medidas de conservación y manejo, según corresponda, para el área de la Convención. La Comisión y los Estados costeros parte o partes concernidos, deberán cooperar en coordinación con sus respectivas medidas de manejo y conservación de acuerdo con el artículo 4 de esta Convención;

ii) Con el consentimiento expreso del Estado costero parte o partes concernido, la Comisión puede establecer de acuerdo con el Anexo III de esta Convención, según corresponda una cuota total de captura o un esfuerzo máximo permisible que aplique a todo el rango de distribución del recurso pesquero; $y$,

iii) En caso que uno o más de los Estados costeros partes no consienta que una cuota total de captura o un esfuerzo pesquero máximo permisible se aplique a todo el rango de distribución del recurso pesquero, la Comisión puede establecer según corresponda, una cuota total de captura o un esfuerzo pesquero máximo permisible que aplique en las áreas de jurisdicción nacional de los Estados costeros parte o partes que sí dieron su consentimiento y en el área de la Convención. El Anexo III se aplicará, mutatis mutandis, al establecimiento de esta cuota total de captura o esfuerzo pesquero máximo permisible por la Comisión (énfasis agregado y traducción nuestra).

Como dijimos, esto fue un logro en solitario de Perú, que prefirió renunciar a que la pesca que había realizado en sus aguas jurisdiccionales se contabilice para la determinación de la cuota, antes que ceder su soberanía y permitir que esas medidas se apliquen en las mismas. Al final, como Perú sostiene que tiene un stock de jurel distinto al stock que es materia de preocupación por parte de la OROP-PS, las autoridades pesqueras con el consejo científico del IMARPE, pueden seguir manejando la pesquería de nuestro stock de jurel de la manera responsable y sostenible como se ha venido haciendo. 